\title{
High Speed Scanning Stereoscopic PIV for 3D Vorticity Measurement in Liquids
}

\author{
Toshio Hori† and Jun Sakakibara $\nmid$ \\ $\dagger$ Institute of Engineering Mechanics and Systems, University of Tsukuba, Tsukuba \\ 305-8573, Japan \\ E-mail: sakakiba@kz.tsukuba.ac.jp
}

\begin{abstract}
A scanning stereo-PIV system was developed to measure the threedimensional distribution of three-component velocity in a turbulent round jet. A laser light sheet produced with a high-repetition-rate YLF pulse laser was scanned by an optical scanner in a direction normal to the sheet. Two high-speed megapixel resolution C-MOS cameras captured the particle images illuminated by the light sheet, and the stereoscopic PIV method was adopted to acquire the 3D-3C-velocity distribution of turbulent water flow. A water jet formed by a round nozzle with an exit diameter of $D=5 \mathrm{~mm}$ was diagnosed by the current technique. The jet Reynolds number was set at $R e \approx 1000$, and the streamwise location of the measurement was fixed at approximately $x=45 \mathrm{D}$. A measurement volume $\left(\sim 100 \times 100 \times 100 \mathrm{~mm}^{3}\right)$ containing 50 velocity planes was scanned in $0.22 \mathrm{~s}$, which was sufficiently short to capture the instantaneous vortical structures. The residue of the continuity equation (divergence) was approximately $7 \%$ of rms vorticity on the centerline of the jet. The iso-vorticity surfaces clearly depict vortical structures in the jet shear layer.

Keywords: Stereo PIV, Three-dimensional measurement, Scanning light sheet, Vortical structure, Turbulence
\end{abstract}

To be published in Meas. Sci. Technol. 


\section{Introduction}

One of the most desirable velocimetries is one that can resolve time-dependent threedimensional three-component velocity vectors in a complex turbulent flow field. Such a method would enable us to obtain all the elements of the velocity gradient tensor, thus allowing the turbulent structures to be visualized by an appropriate vector or scalar such as vorticity or the second invariant of the velocity gradient tensor. Several methods of three-dimensional velocimetry have been presented thus far. Three-dimensional particle tracking velocimetry (3D-PTV), which tracks individual tracer particles using multiple cameras, has been used for several decades (Chiu \& Rib (1956); Nedderman (1961)). Racca and Dewey (1988) measured the 3D velocity of tracer particles seeded in a water flow inside a square duct by using a 16-mm high-speed cine-camera. Within a decade, the film-based camera was replaced by modern CCD cameras as imaging devices for 3DPTV. Nishino et al (1989) developed a 3D-PTV system consisting of three TV cameras. Their Measurements of decaying turbulence in a stirred water tank demonstrated that their technique could be applied to turbulent flows. Since individual particles were identified and tracked with 3D-PTV, the interrogation spot size was relatively smaller than that with regular PIV and thus the technique was robust against the higher shear rate of the flow field. However, each particle has to be sparsely distributed in a measurement volume to identify it in a three-dimensional volume, and the typical number of instantaneous velocity vectors in a volume is on the order of a hundred, which is not sufficient to compute the spatial derivative of velocity. Note that the density of 2D-PTV has been significantly improved (Stitou and Riethmuller (2001)) to the extent, where the three-dimensional location of each particle does not necessarily have to be distinguished.

Particle image velocimetry (PIV) is now commonly used for measuring 2- or 3velocity components in $2 \mathrm{D}$ slices of the velocity field. Since the particles are densely seeded, the number of velocity vectors measured is larger than that with PTV and more feasible for obtaining the spatial derivative of velocity than with PTV. However, it has been difficult to identify thus 3D position of each particle, and thus 3D volumetric measurement has not been available except through holographic PIV (Zhang et al (1997)), which requires a considerably complex optical system. One possible way of extending 2D-PIV to 3D volumetric measurement is with PIV using a fast scanning light sheet. Ushijima and Tanaka (1996) developed a scanning 3D-PIV system that visualizes an entire three-dimensional flow field with scanning laser-light sheets generated from a pair of optical scanners. The technique was applied to rotating fluids accompanied by Ekman boundary layers. Brucker (1995) measured multiple 2D slices of a 2-velocitycomponent with scanning PIV employing one video camera, and evaluated the outof-plane component of velocity by integrating the continuity equation. This method, however, can only be applied in flows for which the third velocity component is known at the boundary of the integration domain. He presented another technique such as scanning stereo PIV, which requires two video cameras viewing the same measurement 
region from different directions to evaluate the out-of-plane component of velocity (Brucker (1996)). He used two regular CCD cameras with a frame rate of $25 \mathrm{~Hz}$ and a scanning laser light sheet, and measured the wake flow behind the spherical cap at $R e=100$. Since the frame rate was low, the slices in the measurement volume were limited to 9 , which was not sufficient for the higher $R e$ flow occupied by the finer scale of eddies.

In the present study, we describe a scanning stereo PIV system capable of higher spatial resolution that resolves eddies in a turbulent flow field at $R e \approx 1000$. Two highspeed mega-pixel resolution C-MOS cameras and a high repetition pulse laser were used to construct the system. Light sheet scanning was achieved with a fast optical scanner. We could measure a fully developed turbulent jet with the system, and were able to visualize $3 \mathrm{D}$ vortical structures.

\section{Method}

It is now common in standard non-scanning stereo PIV for the laser light sheet to be adjusted to fit on the surface of the calibration plate placed on the plane to be measured. Once the calibration procedure was performed on this calibration plate, the projection of the physical location of any point (although limited in view) in the light sheet plane to the image coordinate is known based on the mapping function obtained from the calibration procedure. Similar calibration can be done with scanning PIV such that the calibration plate can be placed on any plane the light sheet illuminates during scanning. However, this method requires considerable time to set the calibration plate on the many planes to be measured. Also, a complicated traversing mechanism is needed to place the calibration plate within the light sheet planes, if they are not parallel to one another as in the present setup. Because of these difficulties, we propose a new method that is suitable for scanning PIV, which we will describe in the sections that follow.

\subsection{Flow Apparatus and Experimental Conditions}

Figure 1 outlines the flow apparatus for a turbulent round jet. Water in the overhead tank was introduced to a honeycomb flow straightener and axisymmetric contraction nozzle having a $5 \mathrm{~mm}$ exit diameter, $D$. The nozzle was figured smoothly in a $3 \mathrm{rd}$ order polynomial curve, and the downstream end of the nozzle was mounted beneath the bottom plate of a 500-mm-high Plexiglas octagonal tank, with $400 \mathrm{~mm}$ between the opposing sidewalls. This configuration created an initially laminar jet with a top hat velocity profile issuing upward into the octagonal tank. The water in the tank overflowed through four equally spaced drainpipes mounted $450 \mathrm{~mm}$ from the bottom surface of the tank. The water was then returned to the overhead tank with a hydro pump. The bulk velocity at the exit of the nozzle was $U_{0}=206 \mathrm{~mm} / \mathrm{s}$, and the corresponding jet Reynolds number was $R e=U_{0} D / \nu=995$, where $\nu$ is kinematic viscosity. A top hat velocity profile at the nozzle exit was achieved as we can see in Fig.2. Since the tank 
had finite dimensions, the flow surrounding the jet had to be reversed. The maximum velocity of the reverse flow in this apparatus was $0.036 U_{m}$, where $U_{m}$ is the center line mean velocity of the jet at each streamwise station.

\subsection{Hardware Setup}

The optical configuration is shown in Fig.3. The laser light beam emitted from a diodepumped Nd:YLF pulse laser (20 mJ per pulse at $2000 \mathrm{~Hz}$, Evolution 30, Positive Light) was converged through a pair of spherical lenses $(f=-150$ and $200 \mathrm{~mm}$ ) and expanded vertically through a cylindrical lens $(f=-70 \mathrm{~mm})$ to form a 2 -mm-thick laser light sheet at the test section. The light sheet was scanned with a flat mirror mounted on an optical scanner (VM2000, GSI Lumonics), which is controlled with a programmable scanner controller (SC2000, GSI Lumonics).

Tracer particles (40 $\mu \mathrm{m}$ Polyamid 12, Daiamid 2157, Daicel Degussa) were seeded in the flow, and scattered light was imaged with two high-speed high-resolution C-MOS cameras (2000 fps with $1024 \times 1024$ pixels resolution in maximum, Fastcam APX, Photron). Camera L was placed at the left of the light sheet plane toward the laser source, and the angle of the camera axis with respect to the light sheet plane was set at approximately $45^{\circ}$. This was also done for camera $R$ at the right. Both cameras could then detect stronger forward scattering light from the particles compared to weaker side or back scattering, with a smaller lens aperture, i.e., a higher f-number of 22 , which provided a greater depth of field.

Each camera was tilted with respect to the lens axis to satisfy the Scheimpflug condition and was focused at the center of the measurement volume. The center of measurement volume $\left(100 \times 100 \times 100 \mathrm{~mm}^{3}\right)$ was located $45 D$ above the nozzle on the jet centerline, which was $800 \mathrm{~mm}$ from the axis of the scanning mirror. Images captured with the cameras were recorded in their memory, and then transferred to a PC hard disk.

Figure 4 is an actual image of particles at the center of the volume. The projected size of a particle was on average approximately 3 pixels at this point, and 4 pixels at the each edge of the measurement volume. Therefore, it was likely that the depth of field would cover the whole measurement volume with this configuration. Our analysis in terms of geometrical optics suggested that the diameter of the most blurred particle located approximately $70 \mathrm{~mm}$ from the plane of focus along the camera axis, reached more than 8 pixels, when the f-number was less than 11. Such significant blurring of particles may make PIV image processing more difficult due to overlapping neighboring particles and reduced image contrast, although we did not systematically evaluate the relationship between error of velocity measurement and the size of the blurred particle.

The calibration plate was made of flat aluminum and had through hole arrays of 1.5 $\mathrm{mm}$ in diameter at $5 \mathrm{~mm}$ intervals to provide the same calibration target pattern on both sides for each camera. The plate was $150 \mathrm{~mm}$ wide, $500 \mathrm{~mm}$ long and $5 \mathrm{~mm}$ thick, and was installed vertically on a servo-motor-driven traversing mechanism (Robo-cylinder, 
IAI) with a traversing accuracy of $20 \mu \mathrm{m}$. The plate was perpendicular to the traversing direction. The Cartesian coordinates with $x$ in the jet's streamwise (vertical) direction, $z$ in the direction normal to the plate, $y$ in the other direction, and the origin at the center of the nozzle exit were defined. Also $u, v$ and $w$ denote $x, y$ and $z$ component of velocity vector, respectively. Note that the rotation axis of the optical scanner was aligned precisely parallel to the $x$-axis.

\subsection{Measurement of Light Sheet Position}

After setting up the imaging hardware, the physical location of the light sheet planes for a given angle of the mirror scanner had to be determined. Figure 5 outlines the geometry of the light sheet and calibration plate. The rotation axis of the laser light sheet, which was parallel to the $x$-axis, was centered at $\left(y_{c}, z_{c}\right)=(1035.8 \mathrm{~mm}, 176.65$ $\mathrm{mm})$ on the $y-z$ plane. The angle of light sheet $\theta$ with respect to the $y$-axis and the angle of scanner mirror $\varphi$ were also defined. Here, the rotation axis of the scanning mirror does not coincide with the rotation axis of the laser light sheet since rays are refracted on the air/Plexiglas/water interface. The equation for the light sheet plane at any given mirror angle was determined in the following way: The scanning mirror was set at angle $\varphi_{A}$, where the light sheet remained at one edge of the measurement volume. The calibration plate was then precisely traversed to position $z=z_{\mathrm{I}}$, where the light sheet hit grid point $P_{\mathrm{I}}$, whose location on the plate was previously established as $\left(x_{\mathrm{I}}, y_{\mathrm{I}}\right)$. Here, the width of the area illuminated by the light sheet was approximately $w_{l}=20 \mathrm{~mm}$, although the light sheet thickness was $t_{l}=2 \mathrm{~mm}$, because the light sheet and the calibration plate were nearly parallel. The center of the illuminated area could be adjusted to point $P_{\mathrm{I}}$ within an error of $\sigma_{y}=1 \mathrm{~mm}$ by operating the traverser. After adjusting the traverser to hit the light sheet on $P_{\mathrm{I}}$, the three-dimensional location of $P_{\mathrm{I}}$, $\left(x_{\mathrm{I}}, y_{\mathrm{I}}, z_{\mathrm{I}}\right)$, was obtained. Here, $z_{\mathrm{I}}$ is the value read from (or written to) the traverser. The error in $z_{\mathrm{I}}$ can be estimated as $t_{l} \sigma_{y} / w_{l}=0.1 \mathrm{~mm}$.

Next, the calibration plate was slightly traversed to another position of $z=z_{\text {II }}$ to hit the light sheet at another point $P_{\mathrm{II}}$, and the point $\left(x_{\mathrm{II}}, y_{\mathrm{II}}, z_{\mathrm{II}}\right)$ was measured in the same manner. This was repeated until 5 different points were measured up to $\left(x_{\mathrm{V}}, y_{\mathrm{V}}, z_{\mathrm{V}}\right)$. Using this set of points, the following equation for this light sheet plane was obtained by the least-squares method;

$$
c_{1}^{A} y+c_{2}^{A} z=1
$$

The same procedure was repeated for another mirror angle $\varphi_{B}$, where the light sheet remained at the other edge of the measurement volume, allowing an equation for the other plane to be obtained.

$$
c_{1}^{B} y+c_{2}^{B} z=1 \text {. }
$$

The angles of these two planes with respect to the $y$-axis are given as

$$
\theta_{A}=\tan ^{-1}\left(-c_{1}^{A} / c_{2}^{A}\right)
$$


and

$$
\theta_{B}=\tan ^{-1}\left(-c_{1}^{B} / c_{2}^{B}\right)
$$

for $\varphi_{A}$ and $\varphi_{B}$, respectively. Thus, the angle of light sheet $\theta$ at a given mirror angle of scanner $\varphi$ was formulated in terms of linear interpolation as

$$
\theta=\frac{\varphi-\varphi_{A}}{\varphi_{B}-\varphi_{A}}\left(\theta_{B}-\theta_{A}\right)+\theta_{A}
$$

Here, the error of $\theta$ associated with the above linear approximation is less than $5.2 \times 10^{-6}$ $\mathrm{rad}$, which is caused by the refraction of the light sheet at the air/Plexiglass/water interface. Finally, the equation for the light sheet plane at any given angle $\theta$ was defined as

$$
c_{1}(\theta) y+c_{2}(\theta) z=1
$$

where the coefficients were calcurated as

$$
\left(\begin{array}{c}
c_{1}(\theta) \\
c_{2}(\theta)
\end{array}\right)=\left(\begin{array}{cc}
\cos \left(\theta-\theta_{A}\right) & -\sin \left(\theta-\theta_{A}\right) \\
\sin \left(\theta-\theta_{A}\right) & \cos \left(\theta-\theta_{A}\right)
\end{array}\right)\left(\begin{array}{c}
c_{1}^{A} \\
c_{2}^{A}
\end{array}\right) .
$$

\subsection{Scanning Mirror Control and Synchronization}

The particle images required to obtain the volumetric velocity distribution at one instance with our scanning stereo PIV consisted of a series of PIV images taken at successive light sheet planes stacked in the azimuthal direction of the light sheet scan. Each PIV image was a pair of particle images separated by time $\Delta t$, and successive light sheet planes were separated in the azimuthal direction by angle $\delta \theta$. Each step in the mirror's angle corresponding to $\delta \theta$ was defined as;

$$
\delta \varphi=\frac{\varphi_{B}-\varphi_{A}}{\theta_{B}-\theta_{A}} \delta \theta
$$

Here, $\Delta t=22 \mathrm{~ms}$ was needed to ensure sufficient particle displacement ( $\sim 10$ pixels $)$ in the images. However, this interval was too short to scan the light sheet completely in the measurement volume due to limitations with the camera's maximum frame rate. However, this interval was too long to leave the light sheet at a single location, resulting in insufficient scanning speed. We thus propose following scanning scheme.

The mirror is placed at initial position $\varphi=\varphi_{0}$, and the laser is emitted. Then, the particle images are captured at time $t=0$. The mirror is then rotated to $\varphi=\varphi_{0}+m \delta \varphi$ at $t=\delta t$, where $m$ is an integer value, and $\delta t$ is the frame interval of the camera, which is identical to the inverse of the camera's frame rate, $f_{c}$. Next, the light sheet is moved to $\varphi_{0}+\delta \varphi$, and continues in a series such as $\varphi_{0}+(m+1) \delta \varphi, \varphi_{0}+2 \delta \varphi, \varphi_{0}+$ $(m+2) \delta \varphi, \ldots, \varphi_{0}+(n-1) \delta \varphi, \varphi_{0}+(n-1+m) \delta \varphi, \varphi_{0}+n \delta \varphi, \varphi_{0}+(n+m) \delta \varphi$, where $n$ denotes the number of measurement planes in a measurement volume. With this motion, the location of the light sheet at any time $t$ is identical to its location at time $t+(2 m-1) \delta t$. The example of the evolution of $\varphi$ in Fig. 6 is referred to in the next paragraph. Thus, the images captured at these two sheet locations, separated by $\Delta t=(2 m-1) \delta t$, were used as a PIV image pair at each measurement plane. This 
allowed more freedom to set $\Delta t$ to an appropriate value independent of the frame rate. We selected $n=50, m=6, \delta \varphi=0.07^{\circ}$, and $\delta \theta=0.11^{\circ}$ for the present study.

To synchronize the optical equipment, trigger pulses were generated by a digital pulse/delay generator (DG535, Stanford Research) to send signals to the cameras, the laser, and the optical scanner. Figure 6 is a timing chart of the signals. Table 1 lists actual settings for the imaging hardware.

A master clock with a frequency of $f_{c}=500 \mathrm{~Hz}$ generated by the digital delay/pulse generator was sent to both cameras to trigger the exposures. Thus, the cameras were operated at a frame rate of 500 fps. A delayed clock, which lagged $1 \mathrm{~ms}$ behind the master clock, was sent to trigger the laser. The signal "Trigger for recording" was manually sent at arbitrary times to the cameras. The cameras actually started recording after this signal was received. This signal and the delayed clock were also sent to the scanner controller, which begins to rotate the mirror to the next angle immediately after every leading edge of the delayed clock, unless the signal "Trigger for recording" was on. Thus, the laser and the cameras were initially running at frequency $f_{c}$, and immediately after manually turning on the "Trigger for recording" signal, image recording and mirror scanning both started. The frame rate of 500 fps was $1 / 4$ of the maximum frame rate of our cameras, since the optical scanner could not realize the expected motion above this frame rate with the present configuration.

Note that the time required to scan the light sheet over the measurement volume became $T_{s}=2(n+m-1) / f_{c}=0.22 \mathrm{~s}$, and also the time required to bring the light sheet back to the initial position was $T_{r}=0.11 \mathrm{~s}$. Thus, the time interval between successive volumetric data was $\Delta T=T_{s}+T_{r}=0.33 \mathrm{~s}$. The spatial interval between adjacent light sheet planes (or measurement planes) was $\Delta z=2.0 \mathrm{~mm}$ at the center of the measurement volume. Thus, the scanning speed was $V_{s}=n \Delta z / T_{s}=454.5 \mathrm{~mm} / \mathrm{s}$. The major parameters of the measurement system are listed in table 2 .

\subsection{Interrogation}

Let us now consider measuring the velocity at a given point $(x, y)$ on a light sheet plane at angle $\theta$. Referring to eq.6, dependent variable $z$ is simply determined by

$$
z=\frac{1-c_{1}^{\theta} y}{c_{2}^{\theta}}
$$

As we can see in Fig.7, particle(s) at $\mathbf{x}=(x, y, z)$ are projected on the 2D image coordinate of the left camera, $\left(X^{L}, Y^{L}\right)=\mathbf{X}^{L}$, and of the right camera, $\left(X^{R}, Y^{R}\right)=\mathbf{X}^{R}$. Both image coordinates were computed with $3 \mathrm{D}-2 \mathrm{D}$ calibration functions, $\mathbf{X}^{L}=$ $\mathbf{F}^{L}(x, y, z)$ and $\mathbf{X}^{R}=\mathbf{F}^{R}(x, y, z)$, which will be described in the following section.

Then, image displacement $\Delta \mathbf{X}^{L}$ and $\Delta \mathbf{X}^{R}$ of particle(s) at $\mathbf{X}^{L}$ and $\mathbf{X}^{R}$ were calculated respectively with regular PIV method in terms of direct cross-correlation between the small sub-domains (interrogation spot) of two successive images separated by time $\Delta t$. Next, the image coordinate of these points for the left camera was 
transformed back into the 3D physical coordinate $(x, y, z)$ in forms,

$$
\begin{aligned}
& \mathbf{x}_{1}^{L}=\mathbf{f}_{1}^{L}\left(\mathbf{X}^{L}\right) \\
& \mathbf{x}_{2}^{L}=\mathbf{f}_{2}^{L}\left(\mathbf{X}^{L}\right) \\
& \mathbf{x}_{1}^{L}+\Delta \mathbf{x}_{1}^{L}=\mathbf{f}_{1}^{L}\left(\mathbf{X}^{L}+\Delta \mathbf{X}^{L}\right) \\
& \mathbf{x}_{2}^{L}+\Delta \mathbf{x}_{2}^{L}=\mathbf{f}_{2}^{L}\left(\mathbf{X}^{L}+\Delta \mathbf{X}^{L}\right) .
\end{aligned}
$$

For the right camera,

$$
\begin{aligned}
& \mathbf{x}_{1}^{R}=\mathbf{f}_{1}^{R}\left(\mathbf{X}^{R}\right) \\
& \mathbf{x}_{2}^{R}=\mathbf{f}_{2}^{R}\left(\mathbf{X}^{R}\right) \\
& \mathbf{x}_{1}^{R}+\Delta \mathbf{x}_{1}^{R}=\mathbf{f}_{1}^{R}\left(\mathbf{X}^{R}+\Delta \mathbf{X}^{R}\right) \\
& \mathbf{x}_{2}^{R}+\Delta \mathbf{x}_{2}^{R}=\mathbf{f}_{2}^{R}\left(\mathbf{X}^{R}+\Delta \mathbf{X}^{R}\right),
\end{aligned}
$$

where $\mathbf{f}$ is the mapping function from the $2 \mathrm{D}$ image coordinate of each camera to an $x-y$ plane at constant $z$ (where the calibration plate was placed) in the 3D physical coordinate system. If the subscripts are 1 , this means $z=z_{1}$. If the subscripts are 2 , this means $z=z_{2}$. The superscript specifies the camera. These mapping functions will also be described in the following section. Let us now calculate point $\mathbf{x}_{t 0}$ where line $\mathbf{x}_{1}^{L} \rightarrow \mathbf{x}_{2}^{L}$ and line $\mathbf{x}_{1}^{R} \rightarrow \mathbf{x}_{2}^{R}$ intersect. Also calculate point $\mathbf{x}_{t 1}$ where line $\mathbf{x}_{1}^{L}+\Delta \mathbf{x}_{1}^{L} \rightarrow \mathbf{x}_{2}^{L}+\Delta \mathbf{x}_{2}^{L}$ and line $\mathbf{x}_{1}^{R}+\Delta \mathbf{x}_{1}^{R} \rightarrow \mathbf{x}_{2}^{R}+\Delta \mathbf{x}_{2}^{R}$ intersect. Since two lines may not intersect due to errors in calibration functions or in measured particle displacement, we define "intersection" as the center of the smallest sphere to which both lines are tangential. Obviously, the two intersecting points, $\mathbf{x}_{t 0}$ and $\mathbf{x}_{t 1}$, are the three-dimensional positions of particle(s) at $t=0$ and $t=\Delta t$, respectively. Thus the velocity vector was obtained by

$$
\mathbf{v}=\left(\mathbf{x}_{t 1}-\mathbf{x}_{t 0}\right) / \Delta t .
$$

The size of the interrogation spot on the image plane of each camera was $28 \times 28$ pixels, which is approximately $2.5 \times 3.5 \mathrm{~mm}^{2}$ in physical space.

\subsection{Calibration}

The calibration functions in the previous sections were obtained with the following procedure. First, the calibration plate was placed at $z=z_{1}$, which is not identical to the position of the plate set in Sec.2.3. The calibration grid images captured by the cameras were processed individually using following algorithms. Each grid point was searched with pattern matching, which found the peak location of the correlation of the original image and template pattern that was similar to the grid. After finding the location of each grid in the image coordinate, the physical location of each grid was determined automatically based on the location of the reference grid and the known spacing between the grids. This procedure produced a table of image coordinates and physical coordinates for the grid points, and created $2 \mathrm{D}-2 \mathrm{D}$ calibration function $\mathbf{f}_{1}^{L}, \mathbf{f}_{1}^{R}$ in the sense of the least squares method. Next, the calibration plate was traversed at $z=z_{2}$, and a similar procedure produced another set of functions $\mathbf{f}_{2}^{L}, \mathbf{f}_{2}^{R}$. The form of 
the calibration function was based on a pinhole camera model with 2nd-order distortion (Willert, 1997), such as

$$
\left(\begin{array}{c}
x \\
y
\end{array}\right)=\mathbf{f}_{\zeta}^{\Pi}(X, Y)=\left(\begin{array}{c}
\frac{a_{0}^{\Pi, \zeta}+a_{1}^{\Pi, \zeta} X+a_{2}^{\Pi, \zeta} Y+a_{3}^{\Pi, \zeta} X^{2}+a_{4}^{\Pi, \zeta} Y^{2}+a_{5}^{\Pi, \zeta} X Y}{1+a_{12}^{\Pi, \zeta} X+a_{13}^{\Pi, \zeta} Y+a_{14}^{\Pi, \zeta} X^{2}+a_{15}^{\Pi, \zeta} Y^{2}+a_{16}^{\Pi, \zeta} X Y} \\
\frac{a_{6}^{\Pi, \zeta}+a_{7}^{\Pi, \zeta} X+a_{8}^{\Pi, \zeta} Y+a_{9}^{\Pi, \zeta} X^{2}+a_{10}^{\Pi, \zeta} Y^{2}+a_{11}^{\Pi, \zeta} X Y}{1+a_{12}^{\Pi, \zeta} X+a_{13}^{\Pi, \zeta} Y+a_{14}^{\Pi, \zeta} X^{2}+a_{15}^{\Pi 1, \zeta} Y^{2}+a_{16}^{\Pi, \zeta} X Y}
\end{array}\right),
$$

where index $\Pi$ denotes either $L$ or $R$ for identifying the camera, and index $\zeta$ denotes 1 if the plate location is $z=z_{1}$ and denotes 2 if the plate location is $z=z_{2}$. Coefficient $a_{i}^{\Pi, \zeta}$ was evaluated with the non-linear least-squares method.

Once these 2D-2D calibration functions were obtained, the remaining 3D-2D functions $\mathbf{F}^{L}$ and $\mathbf{F}^{R}$ were determined in the following way. Point $\mathbf{X}^{\Pi}$ in the image coordinate of camera $\Pi$ is a projection of all points on a line connecting $\mathbf{f}_{1}^{\Pi}\left(\mathbf{X}^{\Pi}\right)$ and $\mathbf{f}_{2}^{\Pi}\left(\mathrm{X}^{\Pi}\right)$. The intersection of this projection line and the measurement plane at angle $\theta$ is given by a function,

$$
\mathbf{x}\left(\theta, \mathbf{X}^{\Pi}\right)=s\left[\mathbf{f}_{2}^{\Pi}\left(\mathbf{X}^{\Pi}\right)-\mathbf{f}_{1}^{\Pi}\left(\mathbf{X}^{\Pi}\right)\right]+\mathbf{f}_{1}^{\Pi}\left(\mathbf{X}^{\boldsymbol{\Pi}}\right)
$$

and

$$
s=\frac{1-c_{1}(\theta) y_{1}-c_{2}(\theta) z_{1}}{c_{1}(\theta)\left(y_{2}-y_{1}\right)+c_{2}(\theta)\left(z_{2}-z_{1}\right)},
$$

where $\left(x_{1}, y_{1}, z_{1}\right)=\mathbf{f}_{1}^{\Pi}\left(\mathbf{X}^{\boldsymbol{\Pi}}\right)$ and $\left(x_{2}, y_{2}, z_{2}\right)=\mathbf{f}_{2}^{\Pi}\left(\mathbf{X}^{\boldsymbol{\Pi}}\right)$. The intersections for all $\theta$ values on which the measurement plane is located are found. Furthermore, these points are found for many possible $\mathbf{X}^{\Pi}$, and finally a set of data with many possible pairs of $\mathbf{X}^{\Pi}$ and $\mathbf{x}\left(\theta, \mathbf{X}^{\Pi}\right)$ can be prepared. This data was fitted into the following function based on a pinhole camera model with 2nd-order distortion using the non-linear least-squares method;

$$
\left(\begin{array}{c}
X \\
Y
\end{array}\right)=\mathbf{F}^{\Pi}\left(\begin{array}{c}
x \\
y \\
z
\end{array}\right)=\left(\begin{array}{c}
\frac{a_{0}^{\Pi}+a_{1}^{\Pi} x+a_{2}^{\Pi} y+a_{3}^{\Pi} z+a_{4}^{\Pi} x^{2}+a_{5}^{\Pi} y^{2}+a_{6}^{\Pi} z^{2}+a_{7}^{\Pi} x y+a_{8}^{\Pi} y z+a_{9}^{\Pi} z x}{1+a_{20}^{\Pi} x+a_{21}^{\Pi} y+a_{22}^{\Pi} z+a_{23}^{\Pi} x^{2}+a_{24}^{\Pi 1} y^{2}+a_{25}^{\Pi} z^{2}+a_{26}^{\Pi} x y+a_{27}^{\Pi} y z+a_{28}^{\Pi} z x} \\
\\
\frac{a_{10}^{\Pi}+a_{11}^{\Pi} x+a_{12}^{\Pi} y+a_{13}^{\Pi} z+a_{14}^{\Pi} x^{2}+a_{15}^{\Pi} y^{2}+a_{16}^{\Pi} z^{2}+a_{17}^{\Pi} x y+a_{18}^{\Pi} y z+a_{19}^{\Pi} z x}{1+a_{20}^{\Pi} x+a_{21}^{\Pi} y+a_{22}^{\Pi} z+a_{23}^{\Pi} x^{2}+a_{24}^{\Pi} y^{2}+a_{25}^{\Pi} z^{2}+a_{26}^{\Pi 1} x y+a_{27}^{\Pi} y z+a_{28}^{\Pi} z x}
\end{array}\right) .
$$

\subsection{Flow chart}

The whole process above is summarized in Fig.8. To begin the procedure, 2D-2D calibration functions were obtained with subprocess (A) (Sec.2.6). Equations of all light sheet planes were estimated in subprocess (B) (Sec.2.3) from their measured positions. Next, 3D-2D calibration functions were obtained by using the equations of light sheet planes and 2D-2D calibration functions in subprocess (C) (Sec.2.6). Finally, velocity vectors were calculated with all the calibration functions and equations of light sheet planes in subprocess (D) (Sec.2.5). 


\section{Error estimation}

Estimation was done to clarify the error in instantaneous velocity and also that in the position of each measurement point. Error factors and values are summarized in table 3. Here the column "Error" denotes the error in each error factors represented by standard deviation of random realizations, and "Sensitivity" denotes the coefficient to adjust the unit of "Error" into millimeters, i.e. "Error in mm" = "Error" $\times$ "Sensitivity".

Although the light sheet position was formulated with eq.1 as a general form, $c_{2}$ is significantly larger than $c_{1}$ because the light sheet plane is mostly perpendicular to the $z$-axis. Thus, the position of the light sheet is now regarded as the $z$ location of a point where it crosses a line $(x, y)=(45 D, 0)$, i.e. the center line of the measurement volume parallel to the $z$ axis. The error in the light sheet position consists of two factors, error in the position of the light sheet at the edges of the measurement volume (Sec.2.3) and error due to presupposing that the angle of the mirror and the angle of the light sheet are linearly related (eq.5). The first factor can be further divided into another two. The first is the error in the location of $P_{\mathrm{I}} \ldots P_{\mathrm{V}}$ equivalent to the error of $z_{\mathrm{I}} \ldots z_{\mathrm{V}}$, which is estimated as $0.1 \mathrm{~mm}$ as described in Sec.2.3. The second is the error in the servomotordriven traversing mechanism, which is expected to be $0.02 \mathrm{~mm}$. The error associated with the linear approximation of the light sheet angle to the mirror angle was $5.2 \times 10^{-6}$ $\mathrm{rad}$, as stated in Sec.2.3. This leads to error in the light sheet position of $0.0054 \mathrm{~mm}$.

The calibration function is also a source of position error. The error in the 2D-2D calibration function represented by eq.13 was estimated by,

$$
\sigma_{2 D}=\left(\frac{1}{n} \sum_{i=1}^{n}\left(f_{y}\left(X_{i}, Y_{i}\right)-y_{i}\right)^{2}\right)^{1 / 2},
$$

where $f_{y}$ denotes a $y$ component of function $\mathbf{f}$, and $i$ is the index of the grid point in the calibration plate. This might include fitting error in the function to the grid point in the calibration image, and also machining error in the grid position, which is expected to be $0.01-0.05 \mathrm{~mm}$. The value of $\sigma_{2 D}$ was $0.05 \mathrm{~mm}$. Note that this calibration error was comparable to the error in the 3 rd order polynomial type calibration function. The traversing error in the calibration plate was $0.02 \mathrm{~mm}$.

The error in 3D-2D calibration function (eq.16) was estimated by,

$$
\sigma_{3 D}=\left(\frac{1}{n} \sum_{i=1}^{n}\left(F_{X}\left(x_{i}, y_{i}, z_{i}\right)-X_{i}\right)^{2}\right)^{1 / 2}
$$

where $F_{X}$ denotes an $X$ component of function $\mathbf{F}$, and $i$ is the index of the grid point in the calibration plate placed in the middle of $z_{1}$ and $z_{2}$. The value of $\sigma_{3 D}$ was 0.05 $\mathrm{mm}$. Thus, the squared summation of these errors is $0.13 \mathrm{~mm}$ as standard deviation type total position error.

Next, let us consider the error in velocity measurement. Here, we have only described the error in velocity component $v$ with $u=w=0$ for the sake of simplicity. Note that the error in $v$ is expected to be similar to the error in $w$, and larger than the 
error in $u$. Since the error in velocity may depend on its magnitude, that listed in table 3 is $v=U_{m}(=24 \mathrm{~mm} / \mathrm{s})$. The velocity error has two major factors. The first is the error in measured particle displacement on the image. This is often called "sub-pixel error", and was typically estimated as 0.1 pixels in our own simulations. A similar degree of error independently arose in both camera images.

The second factor is the error in the spatial gradient of the 2D-2D calibration function eq.13. The measured velocity $v_{\text {meas }}$ at image coordinate $\left(X_{i}, Y_{i}\right)$, on which the location of the $i$-th grid of calibration plate $\left(x_{i}, y_{i}\right)$ is projected, is approximated as

$$
\left.v_{\text {meas }} \approx \frac{\Delta X}{\Delta t} \frac{\partial f_{y}(X, Y)}{\partial X}\right|_{X=X_{i}, Y=Y_{i}},
$$

where $\Delta X$ is the $X$ component of particle displacement in the image. In the meantime, "true" velocity was approximated by

$$
v_{\text {true }}=\left.\frac{\Delta X}{\Delta t} \frac{\partial y}{\partial X}\right|_{y=y_{i}} \approx \frac{\Delta X}{\Delta t} \frac{y_{i}-y_{j}}{X_{i}-X_{j}},
$$

where $j$ denotes the index of a grid that is the neighboring grid of the $i$-th grid facing the $y$ direction. Thus, the error in $v_{\text {meas }}$ was estimated by subtracting $v_{\text {true }}$

$$
\begin{aligned}
\sigma_{v} & =\left(\frac{1}{n} \sum_{i=1}^{n}\left(v_{\text {meas }}-v_{\text {true }}\right)^{2}\right)^{1 / 2} \\
& =\frac{\Delta X}{\Delta t}\left(\frac{1}{n} \sum_{i=1}^{n}\left(\left.\frac{\partial f_{y}(X, Y)}{\partial X}\right|_{X=X_{i}, Y=Y_{i}}-\frac{y_{i}-y_{j}}{X_{i}-X_{j}}\right)^{2}\right)^{1 / 2}
\end{aligned}
$$

This was computed from actual calibration images and the standard deviation is listed in table 3. Note that the approximated value of $v_{\text {true }}$ includes gradient of randomly occurring machining error in the actual grid spacing of the calibration plate $(0.02 \mathrm{~mm}$ - $0.05 \mathrm{~mm}$ ) and also the error in the pattern matching process to measure the grid location on the calibration image. Thus the value of $\sigma_{v}$ may have been overestimated. The ground total of error in velocity measurement was $0.85 \mathrm{~mm} / \mathrm{s}\left(0.035 U_{m}\right)$ in standard deviation.

\section{Results}

Let us begin our discussion on the measured data with the mean and RMS velocity profile in Fig.9. Here, $r$ is the radial distance from the jet's centerline estimated by fitting a 2D Gaussian function on the mean velocity data. The mean and RMS velocity were obtained by ensemble-averaging over the 8 independent series of velocity data: each series consists of 12 instantaneous volumetric velocity data measured with $0.33 \mathrm{~s}$ intervals. The profile was then computed by averaging in the azimuthal direction, and also by averaging in the streamwise direction after normalizing with the half velocity width, $b$, and centerline mean velocity, $U_{m}$, at each streamwise station. Both mean and RMS velocity profiles had reasonable agreement with the data previously measured by 
Wygnanski and Fiedler (1969). Note that the centerline mean velocity at $x=45 D$ was $U_{m}=24 \mathrm{~mm} / \mathrm{s}$ and the velocity half width was $b=22 \mathrm{~mm}$.

The three-dimensional velocity vectors have been reconstructed in Fig.10. Here, only a few sections of the measurement domain have been displayed for clarity. The centerline of the jet is located near the center of the domain. The velocity vectors are raw results and no smoothing or enhancement has been done.

The vorticity vectors were computed with the 2 nd order finite difference of the velocity vectors and were smoothed by averaging $3 \times 3 \times 3$ nodes surrounding each node to reduce random noise. Since the original measurement grids (nodes) were not parallel because the light sheet planes were not, they were relocated to form parallel grids with a linear interpolation scheme prior to calculating vorticity. Figure 11 represents surfaces of constant vorticity magnitude, $|\omega|=2.5 \mathrm{~s}^{-1}$. Vortical structures exhibiting elongated loops can clearly be observed.

These vortical structures convect downstream without significantly changing their geometrical features. This implies that the scanning duration $T_{s}(=0.22 \mathrm{~s})$ and interval of successive volumes $\Delta T(=0.33 \mathrm{~s})$ are sufficiently small to capture the temporal evolution of such structures.

Quantities derived from velocity in the $y-z$ cross-section at $x=45 D$ represented by symbol A in Fig.10 are depicted in Fig.12. Figure 12(a) shows the in-plane velocity component (vector) and the out-of-plane velocity component (contour). The direction in which the light sheet is scanned is indicated by the arrows at the right of the figure. Vectors and contours in each corner of the measurement domain are eliminated where particles are not projected onto either of the CCD cameras. The jet core centered near $y=z=0$ is dominated by the large out-of-plane velocity component with complex fine eddies.

Figure 12(b) and (c) depict corresponding vorticity distributions and the residue of the continuity equation, which should be zero in theory. Vorticity and residue peaks are concentrated around the center of the plane. It is obvious that the instantaneous residue is much smaller than the vorticity. Figure 13 plots the ensemble RMS vorticity magnitude and ensemble RMS residue of the continuity equation. Both quantities were also square-summed over the $x$ direction within the measurement volume. The residue has a maximum at $r=0$, where the vorticity is also largest. The ratio between RMS vorticity and RMS residue of the continuity equation at $r=0$ was approximately 0.07 . The residue was reasonably small to capture the vortical structures.

\section{Discussion}

In this section, we discuss the time and spatial scale of the flow field, and temporal and spatial resolution with the present technique.

Since the light sheet scan in the measurement volume takes a finite time, $T_{s}$, the measured vortical structure may be distorted compared with a truly instantaneous structure. The greatest distortion is estimated with the ratio of the convection velocity 
of the structure and the scanning speed, $V_{s}(=454.5 \mathrm{~mm} / \mathrm{s})$. Since the convection velocity of the vortical structures are approximately the same as the local mean velocity (Wygnanski and Fiedler, 1969), the maximum convection velocity can be assumed to be $U_{m}$. Thus, the largest distortion is estimated as $U_{m} / V_{s}=0.053$, which is sufficiently small to discuss the topology of the vortical structures.

The time scale of the smallest eddies in the turbulent flow field is represented with a Kolmorogov time scale, $\tau=(\nu / \epsilon)^{1 / 2}$, where $\epsilon$ is the dissipation rate. The Kolmogorov time scale of the present jet is estimated as $\tau=0.028 \mathrm{~s}$ in the measurement volume, where $\epsilon$ was evaluated based on the value reported by Wygnanski and Fiedler (1969). It is apparent that the scanning duration, $T_{s}$, is an order larger than the Kolmogorov scale. However, the time scale of the large eddies, $\tau_{L}=b / U_{m}$, is approximately $0.9 \mathrm{~s}$ which is meaningfully larger than $T_{s}$. At this point, we can conclude that the temporal resolution is too low to resolve the smallest eddies, but high enough to resolve the large coherent vortices, such as the looped structures in Fig.11.

In addition to the time scale, the spatial scale should also be considered. The Kolmogorov length scale is estimated as $\eta=\left(\nu^{3} / \epsilon\right)^{1 / 4}=0.17 \mathrm{~mm}$. Although direct numerical simulation typically requires a grid spacing approximately 2-3 times wider than the Kolmogorov length scale, the interrogation spot size $(\approx 2 \mathrm{~mm})$ is still too large to resolve the smallest eddies. Furthermore, as previously mentioned, the vorticity was computed by averaging over surrounding $3 \times 3 \times 3$ nodes, i.e. the spatial resolution of vorticity is roughly $6 \mathrm{~mm}$. Thus, the magnitude of measured vorticity may have significant error due to the lack of spatial resolution. However, the residue of the continuity equation is sufficiently small as can be seen from Fig.13. This implies that the measured vorticity field can be viewed as a spatially low-pass-filtered field that can still satisfy continuity, and the topology and dynamics of large-scale structures can be discussed on the basis of such a low-pass-filtered field without sub-grid small scales.

\section{Conclusion}

We developed scanning stereo-PIV system to measure the three-dimensional distribution of three-component velocity in a turbulent round jet. A laser light sheet produced with a high-repetition-rate YLF pulse laser was scanned with an optical scanner. The evolution of the mirror angle had the form of a linear slope with small amplitude oscillations, which allowed more freedom in choosing the time interval $\Delta t$ of a pair of particle images. Two high-speed mega-pixel resolution C-MOS cameras captured the particle images illuminated by the light sheet, and we adopted new stereoscopic PIV method to acquire the 3D-3C-velocity distribution of a turbulent water jet that was formed by a round nozzle with an exit diameter of $D=5 \mathrm{~mm}$. The jet Reynolds number was set at $R e \approx 1000$, and the streamwise location of the center of measurement volume was fixed at approximately $x=45 \mathrm{D}$, where the centerline mean velocity was approximately 24 $\mathrm{mm} / \mathrm{s}$.

The measurement volume $\left(\sim 100 \times 100 \times 100 \mathrm{~mm}^{3}\right)$ containing 50 velocity planes 
was scanned in $0.22 \mathrm{~s}$, which was sufficiently short to capture instantaneous vortical structures. The residue of the continuity equation (divergence) was approximately $7 \%$ of rms vorticity on the centerline of the jet. Iso-vorticity surfaces clearly showed largescale coherent vortices.

\section{Acknowledgments}

We are grateful to Prof. H. Hayami of Kyusyu University, the leader of Dynamic PIV Project, for his cooperation and Dr. S. Aramaki of the same university for his assistance with the experiments. We also wish to thank the project members, Dr. K. Okamoto of Tokyo University, Dr. K. Nishino of Yokohama National University, Dr. M. Kawahashi of Saitama University, and Dr. T. Kobayashi of the Japan Automobile Research Institute for their helpful discussions. The experiments were conducted at the Institute for Materials Chemistry and Engineering of Kyushu University, Japan. This work was supported by a Grant-in-Aid (No. 14205031) for Scientific Research by Japan Society of Promotion for Science.

\section{References}

Brucker C 1995 Digital-Particle-Image-Velocimetry (DPIV) in a scanning light-sheet: 3-D starting flow around a short cylinder Exp. Fluids 19 255-63

-1996 3-D Scanning-Particle-Image-Velocimetry: Technique and Application to a Spherical Cap Wake Flow Applied Scientific Research 56 157-79

Chiu W C and Rib L N 1956 The rate of dissipation of energy and the energy spectrum in a low-speed turbulent jet Trans. Am. Geophys. Union 37 13-26

Zhang J, Tao B and Katz J 1997 Turbulent flow measurement in a square duct with hybrid holographic PIV Exp. Fluids 23 373-381

Nedderman R M 1961 The use of stereoscopic photography for the measurement of velocities in liquids Chem. Eng. Sci. 16 113-9

Nishino K, Kasagi N and Hirata M 1989 Three-dimensional particle tracking velocimetry based on automated digital image processing Trans. ASME, J.Fluids Eng. 111 384-91

Recca R G and Dewey J M 1988 A method for automatic particle tracking in a three-dimensional flow fluid Exp. Fluids 6 25-32

Stitou A and Riethmuller M L 2001 Extension of PIV to super resolution using PTV Meas. Sci. Technol. 12 1398-1403

Ushijima S and Tanaka N 1996 Three dimensional particle tracking velocimetry with laser light sheet scannings Trans. ASME J.Fluids Eng. 118 352-7

Willert C 1997 Stereoscopic digital particle image velocimetry for application in wind tunnel flows Meas. Sci. Technol. 8 1465-79

Wygnanski I and Fiedler H 1969 Some measurements in the self-preserving jet J. Fluid Mech. 38 $577-612$ 


\section{Tables and table captions}

Table 1. Operating conditions for imaging hardware.

\begin{tabular}{l|l}
\hline Hardware & Operating conditions \\
\hline Pulse/Delay generator & $500 \mathrm{~Hz}$ master pulse with $1 \mathrm{~ms}$ delayed pulse \\
C-MOS camera & $500 \mathrm{fps}, 1024 \times 1024$ pixels \\
Nd:YLF pulse laser & $500 \mathrm{~Hz}^{*}, 20 \mathrm{~mJ} /$ pulse \\
Optical scanner & $500 \mathrm{~Hz}^{*}, 0.14_{p-p}^{\circ}$ \\
\hline
\end{tabular}

* Synchronize with delayed pulse.

Table 2. System parameters

\begin{tabular}{l|l}
\hline Description & value \\
\hline Number of planes in a volume, $n$ & 50 \\
Light sheet thickness, $t_{l}$ & $2 \mathrm{~mm}$ \\
Interval between adjacent planes, $\Delta z$ & $2 \mathrm{~mm}$ \\
Scanning time for a volume, $T_{s}$ & $0.22 \mathrm{~s}$ \\
Time interval between successive volumes, $\Delta T$ & $0.33 \mathrm{~s}$ \\
Interrogation spot size & $28 \times 28$ pixels $\left(2.5 \times 3.5 \mathrm{~mm}^{2}\right)$ \\
Size of measurement volume & $100 \times 100 \times 100 \mathrm{~mm}^{3}$ \\
\hline
\end{tabular}

Table 3. Major sources of error in measurement

\begin{tabular}{|l|l|l|r|r|r|}
\hline \multicolumn{5}{|c|}{ Position } \\
\hline \multirow{3}{*}{ Light sheet } & Edge & $P$ & Error & Sensitivity & Error in mm \\
\cline { 2 - 5 } & & Traverser & $0.1 \mathrm{~mm}$ & 1 & $0.1 \mathrm{~mm}$ \\
& Relation between $\varphi$ and $\theta$ & $5.2 \times 10^{-6} \mathrm{rad}$ & $1035.8 \mathrm{~mm}$ & $0.0054 \mathrm{~mm}$ \\
\hline \multirow{3}{*}{ Calibration } & $2 \mathrm{D}-2 \mathrm{D}$ & Fitting+machining & $0.05 \mathrm{~mm}$ & 1 & $0.05 \mathrm{~mm}$ \\
Functions & Traverser & $0.02 \mathrm{~mm}$ & 1 & $0.02 \mathrm{~mm}$ \\
\cline { 2 - 5 } & 3D-2D & Fitting+machining & $0.05 \mathrm{~mm}$ & 1 & $0.05 \mathrm{~mm}$ \\
& Traverser & $0.02 \mathrm{~mm}$ & 1 & $0.02 \mathrm{~mm}$ \\
\hline \multicolumn{4}{|c|}{ Total } & & $0.13 \mathrm{~mm}$ \\
\hline
\end{tabular}

\begin{tabular}{|c|c|c|r|}
\hline \multicolumn{4}{|c|}{ Velocity } \\
\hline Source & Error & Sensitivity & Error in mm/s \\
\hline Particle displacement (Camera L) & 0.1 pixels & $4.2 \mathrm{~mm} /($ pixel·s) & $0.42 \mathrm{~mm} / \mathrm{s}$ \\
Particle displacement (Camera R) & 0.1 pixels & $4.2 \mathrm{~mm} /($ pixel $\cdot \mathrm{s})$ & $0.42 \mathrm{~mm} / \mathrm{s}$ \\
\hline Calibration (Camera L) & $0.018 \%$ & $24 \mathrm{~mm} / \mathrm{s}$ & $0.43 \mathrm{~mm} / \mathrm{s}$ \\
Calibration (Camera R) & $0.018 \%$ & $24 \mathrm{~mm} / \mathrm{s}$ & $0.43 \mathrm{~mm} / \mathrm{s}$ \\
\hline \multicolumn{3}{|c|}{ Total } & $0.85 \mathrm{~mm} / \mathrm{s}$ \\
\hline
\end{tabular}




\section{Figure captions}

Figure 1. Flow apparatus.

Figure 2. Velocity profile at $0.25 D$ behind jet exit. Velocity is normalized by velocity at center of jet.

Figure 3. Optical arrangement.

Figure 4. Actual image of particles captured by camera L in Fig.3.

Figure 5. Schematic of light sheet plane viewed from top.

Figure 6. Time chart for signals and operation. $m$ is 3 in this time chart for simplicity, although actual $m$ was set at 6 .

Figure 7. Projection of particle(s) onto camera. Light sheet plane (thick gray line) and calibration planes are also indicated.

Figure 8. Flowchart for data processing procedure. 
Figure 9. Mean and RMS velocity profiles.

Figure 10. Three-dimensional view of instantaneous velocity vectors. Tic intervals are $10 \mathrm{~mm}$ in all directions. Symbol A represents $x$-location of cross-sections in Fig. 12 .

Figure 11. Surfaces of constant vorticity magnitude, $|\omega|=2.5 \mathrm{~s}^{-1}$. Orientation of axis and tic intervals are identical to that in Fig.10. (a) $t=t_{0}$, (b) $t=t_{0}+0.330 \mathrm{~s}$, (c) $t=t_{0}+0.660 \mathrm{~s}$

Figure 12. Instantaneous quantities on $y-z$ plane at $x$-location represented by symbol A in Fig.10; (a) In-plane velocity component (vectors) and out-of-plane velocity component (contours); (b) $x$-component of vorticity; (c) Residue of continuity equation.

Figure 13. Ensemble RMS vorticity magnitude and ensemble RMS residue of continuity equation. Both quantities were also square-summed over $x$ direction within measurement volume. 
Fig.1

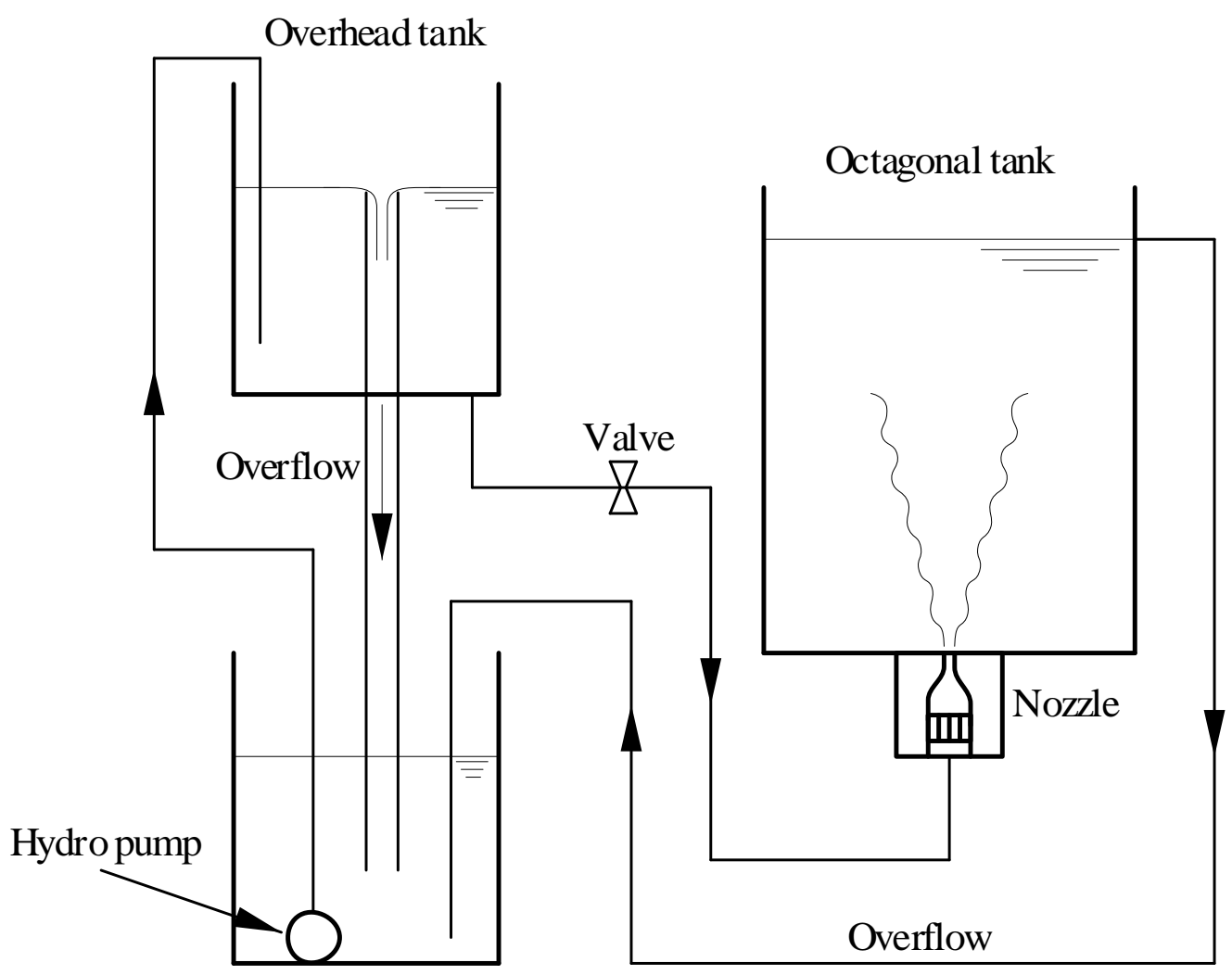

Fig.2

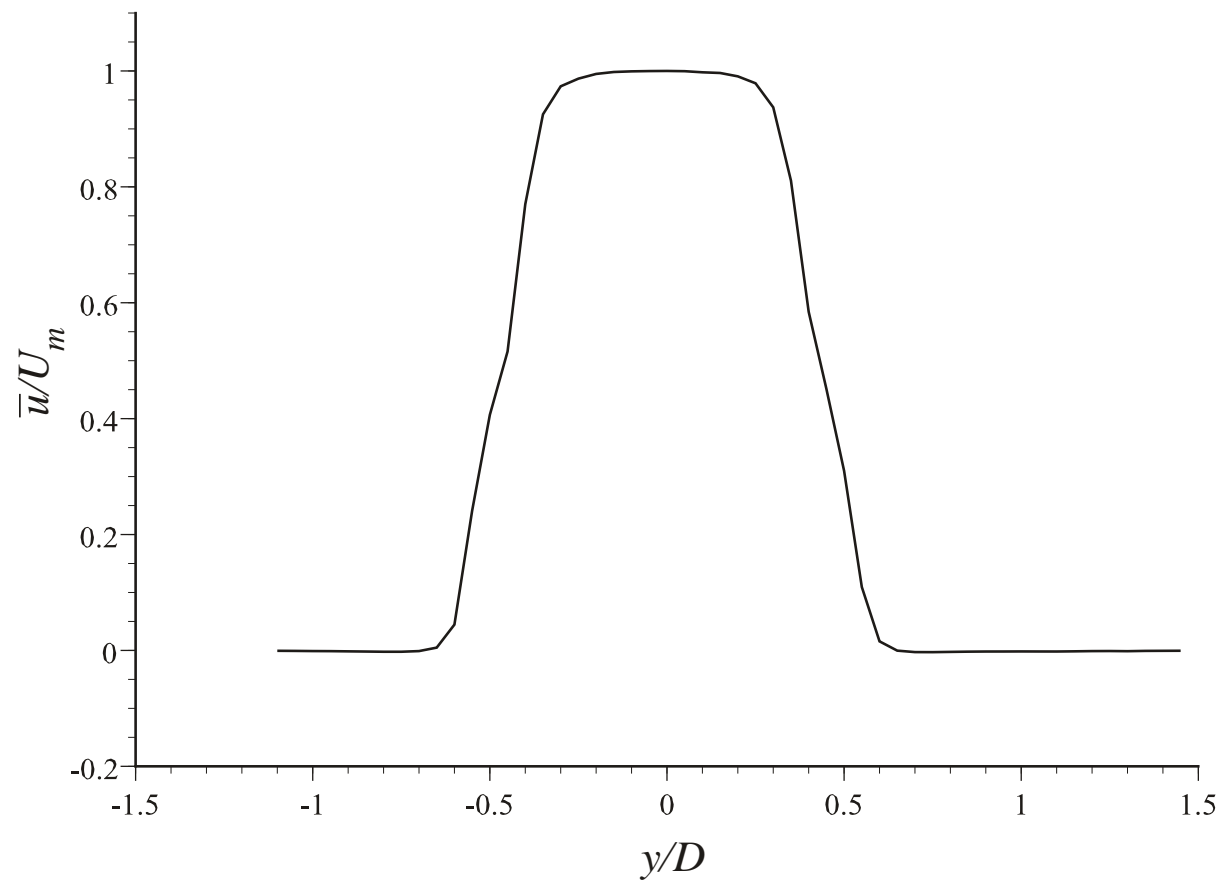


Fig.3
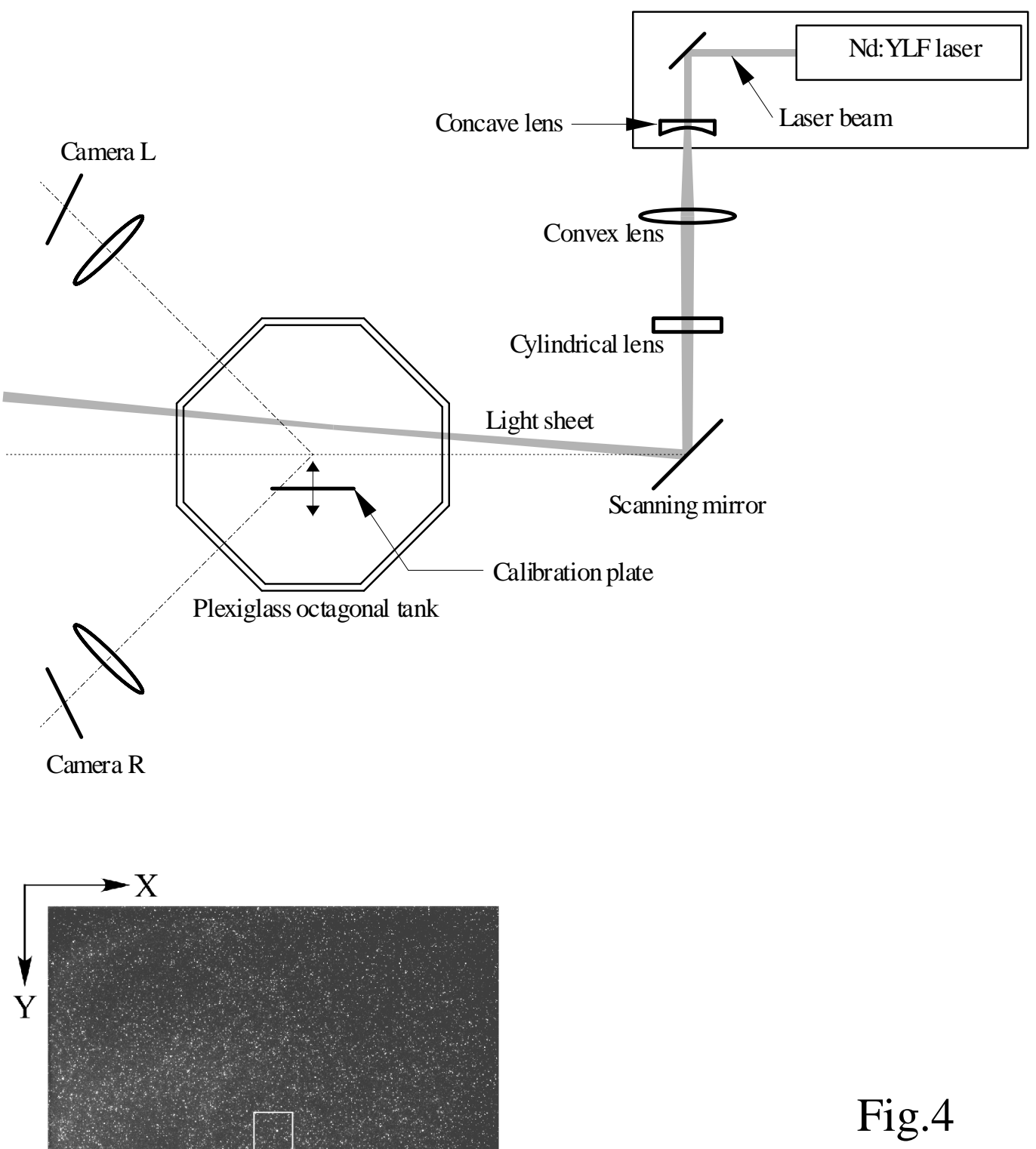

Streamwise

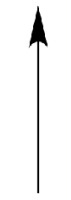


Fig.5

Fig.6
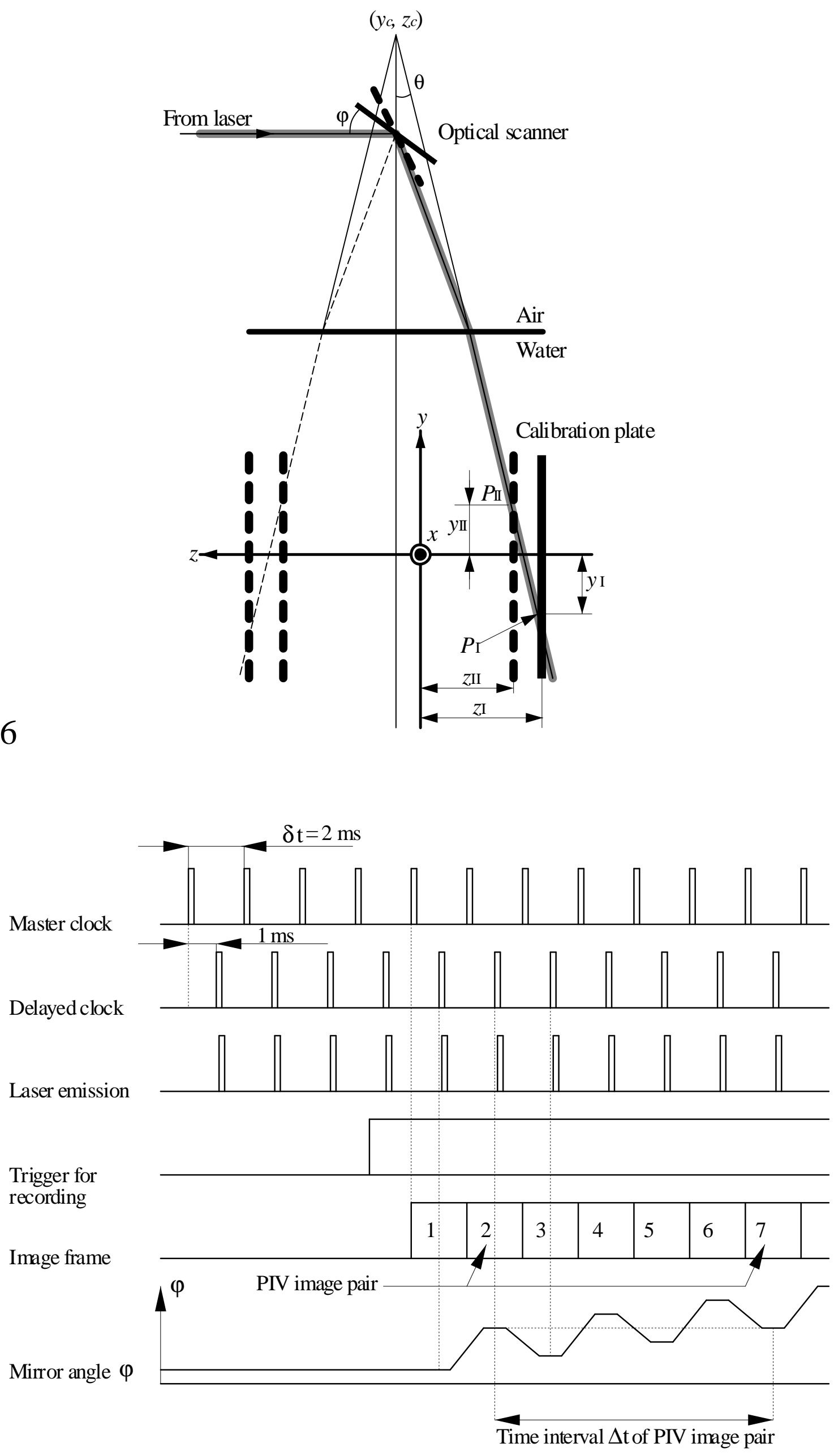
Fig.7

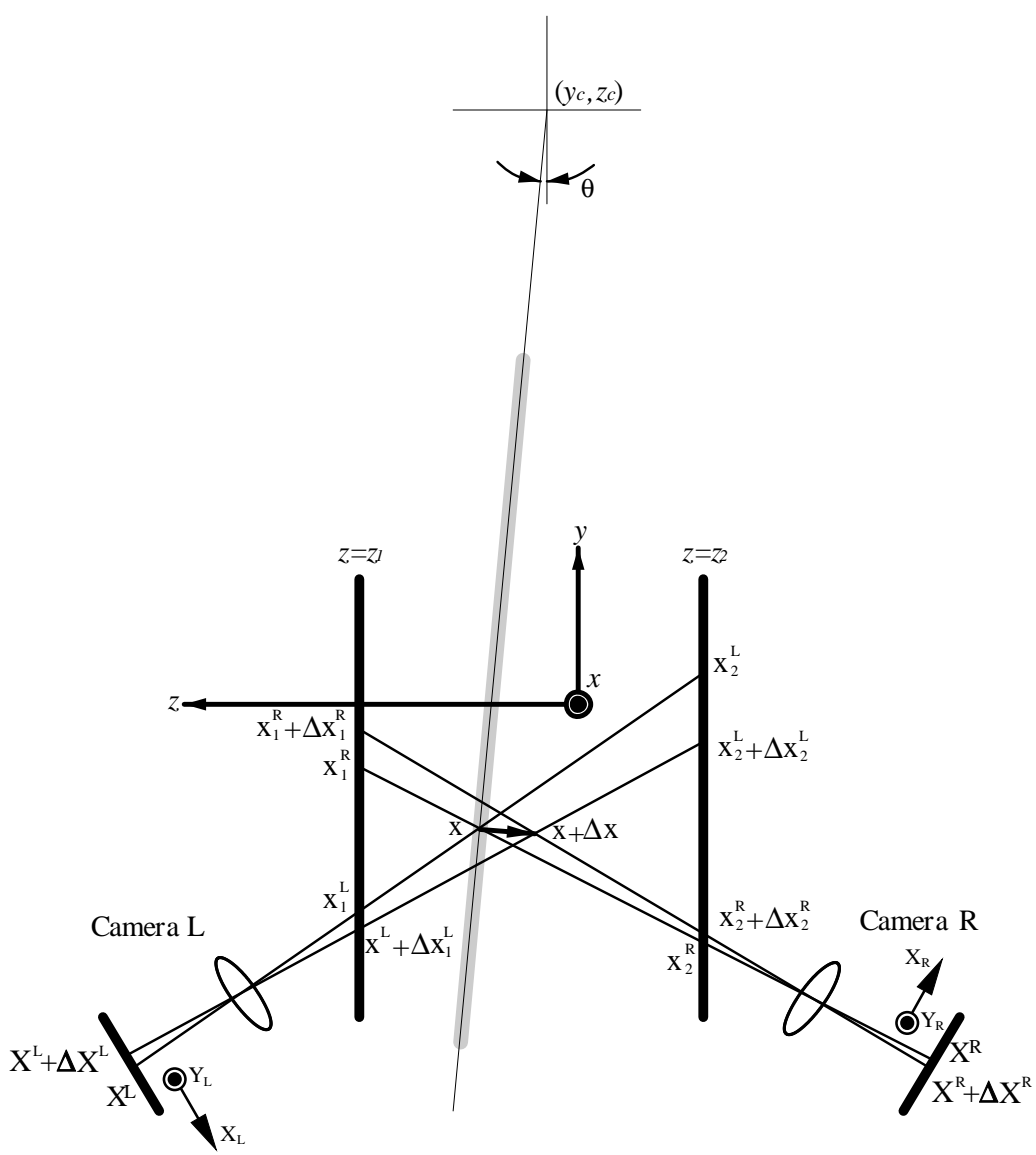

Fig.8

(A)

(B)

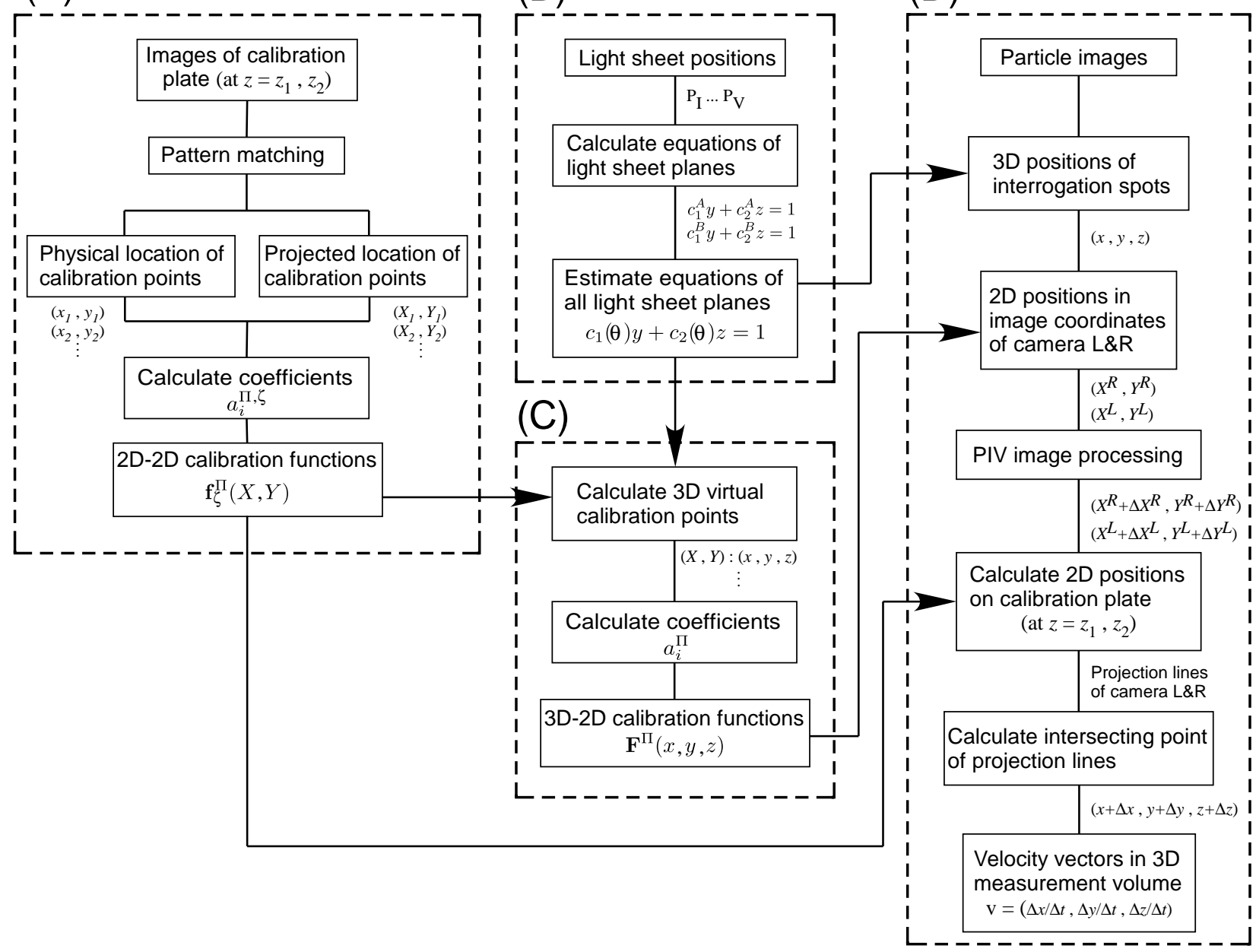


(a)

Fig.9

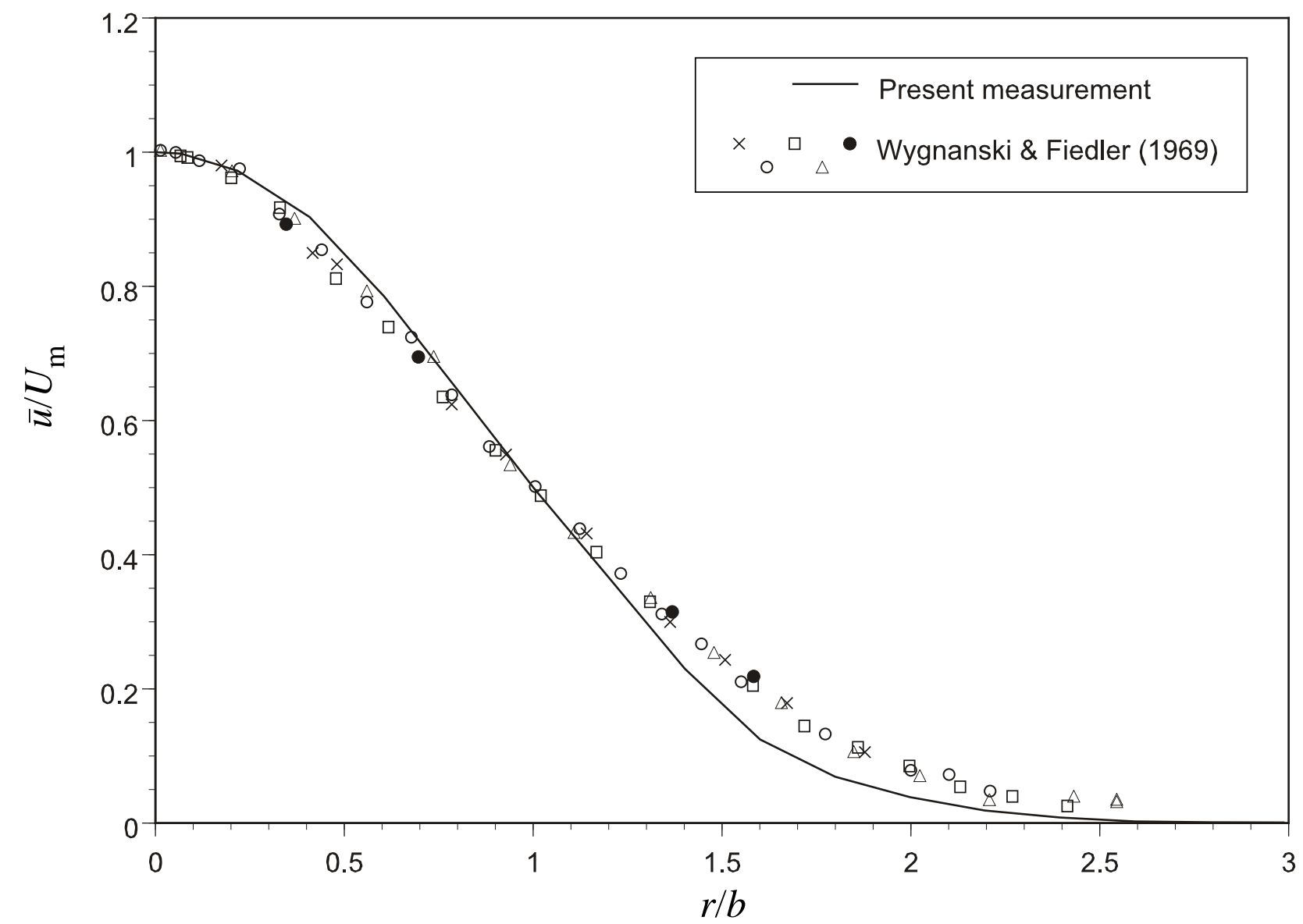

(b)

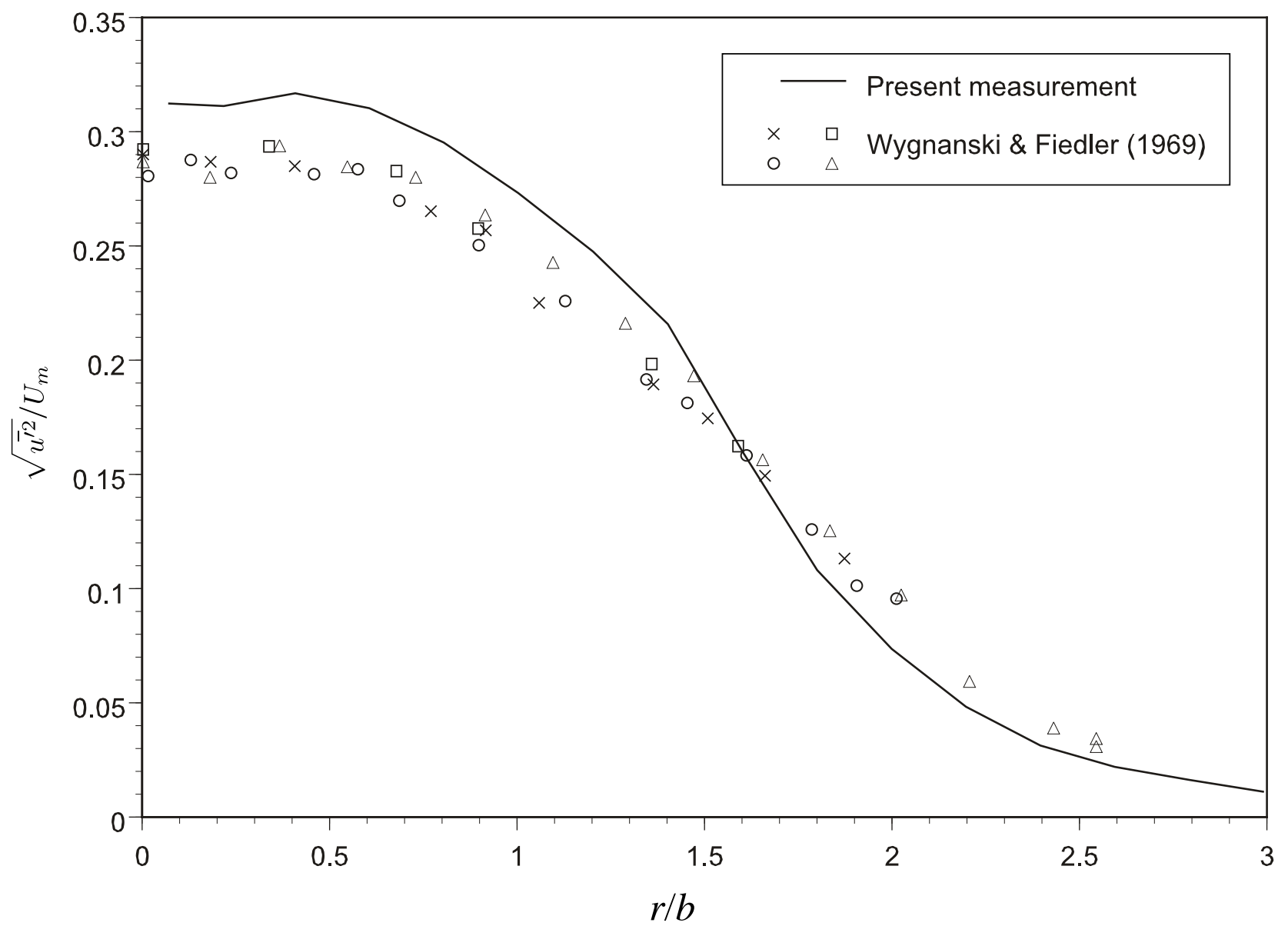


Fig.10

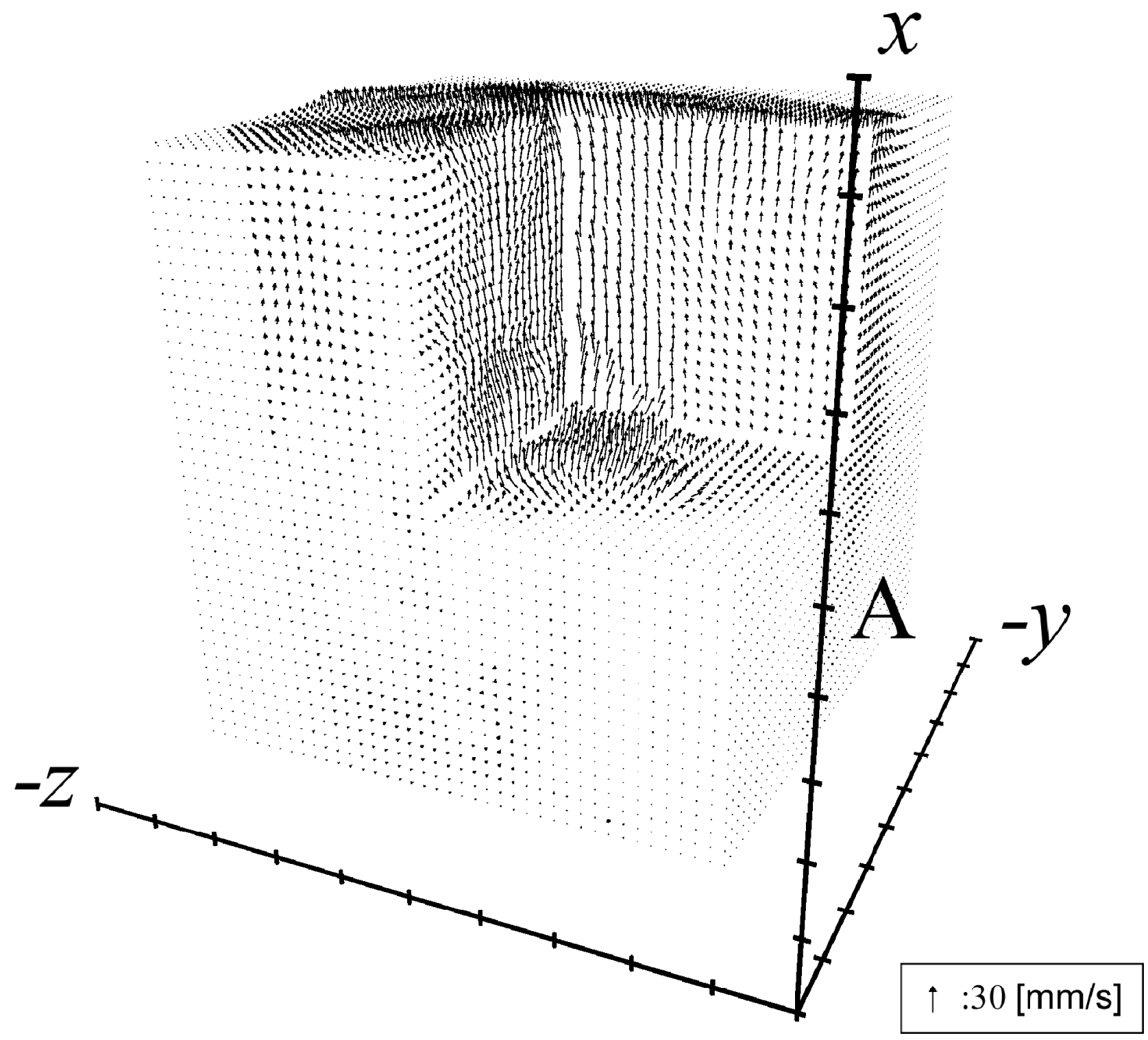


Fig.11
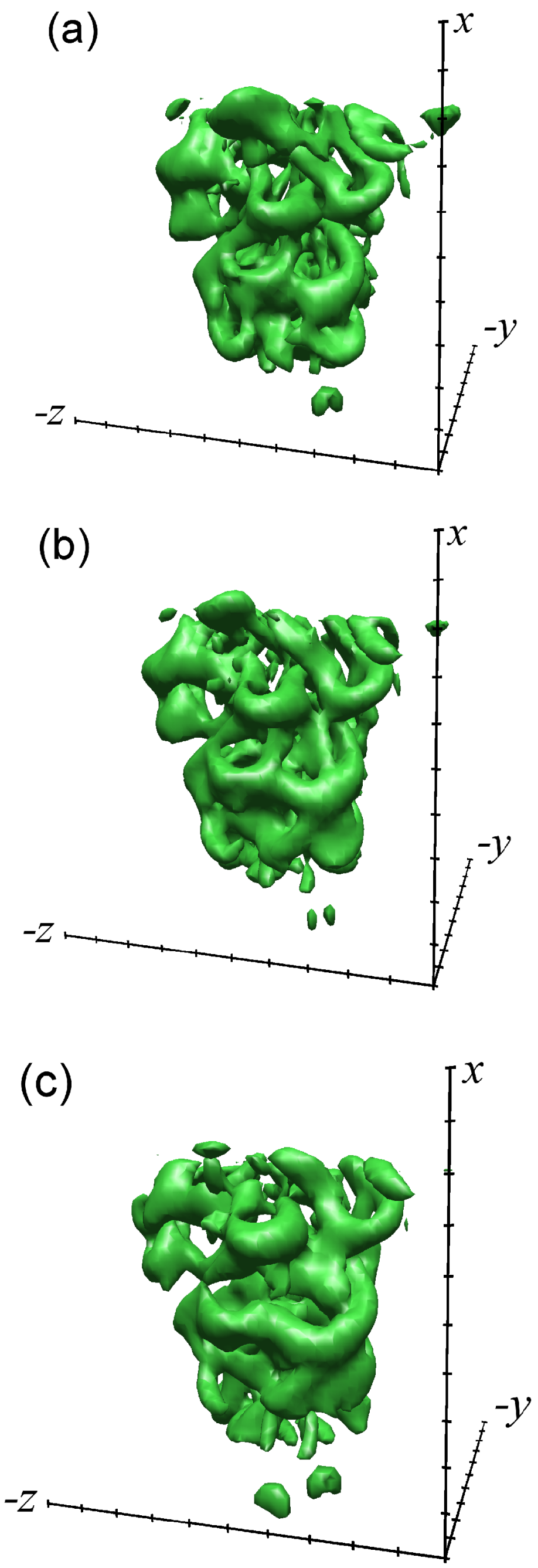
Fig.12

(a)

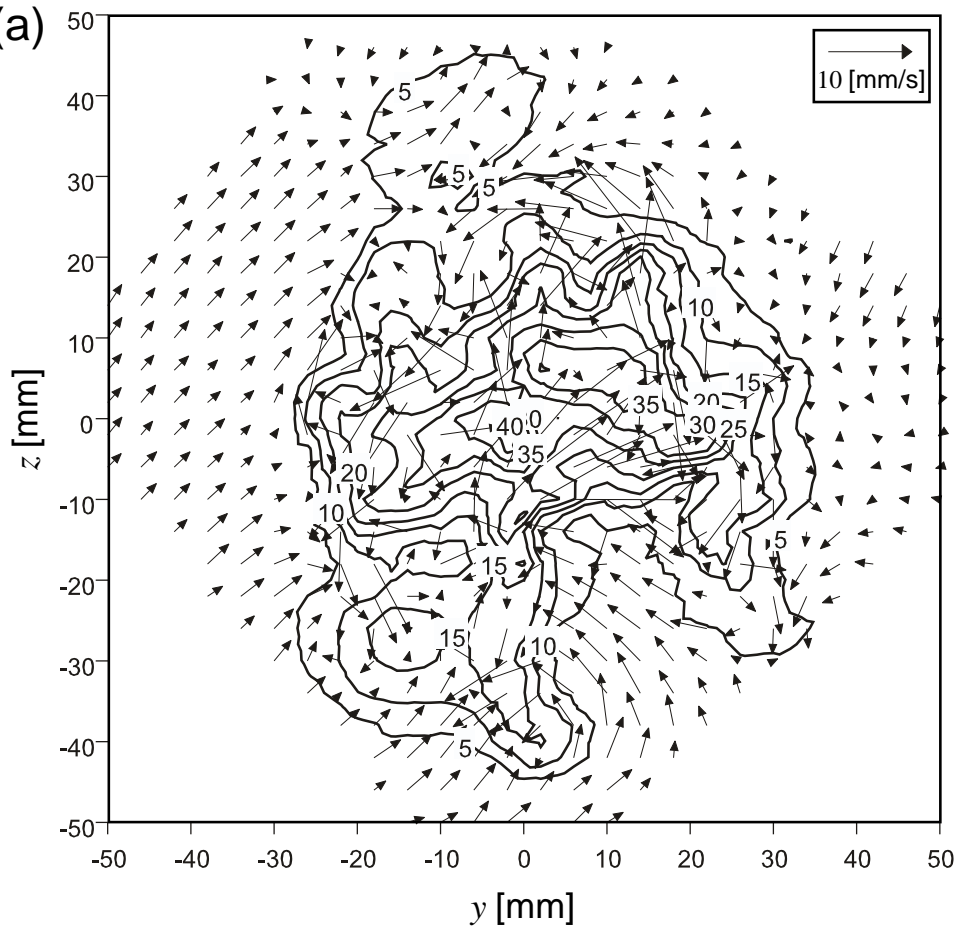

Scan direction
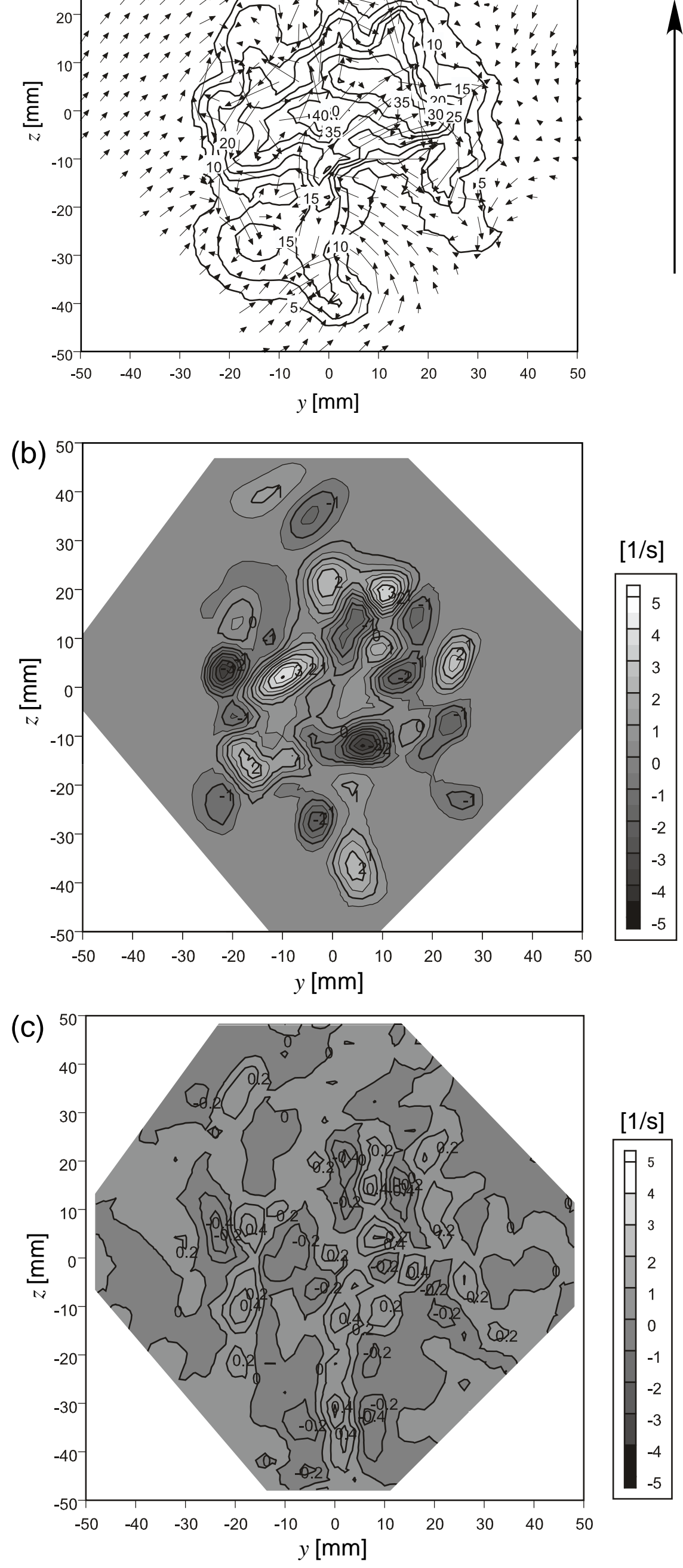
Fig.13

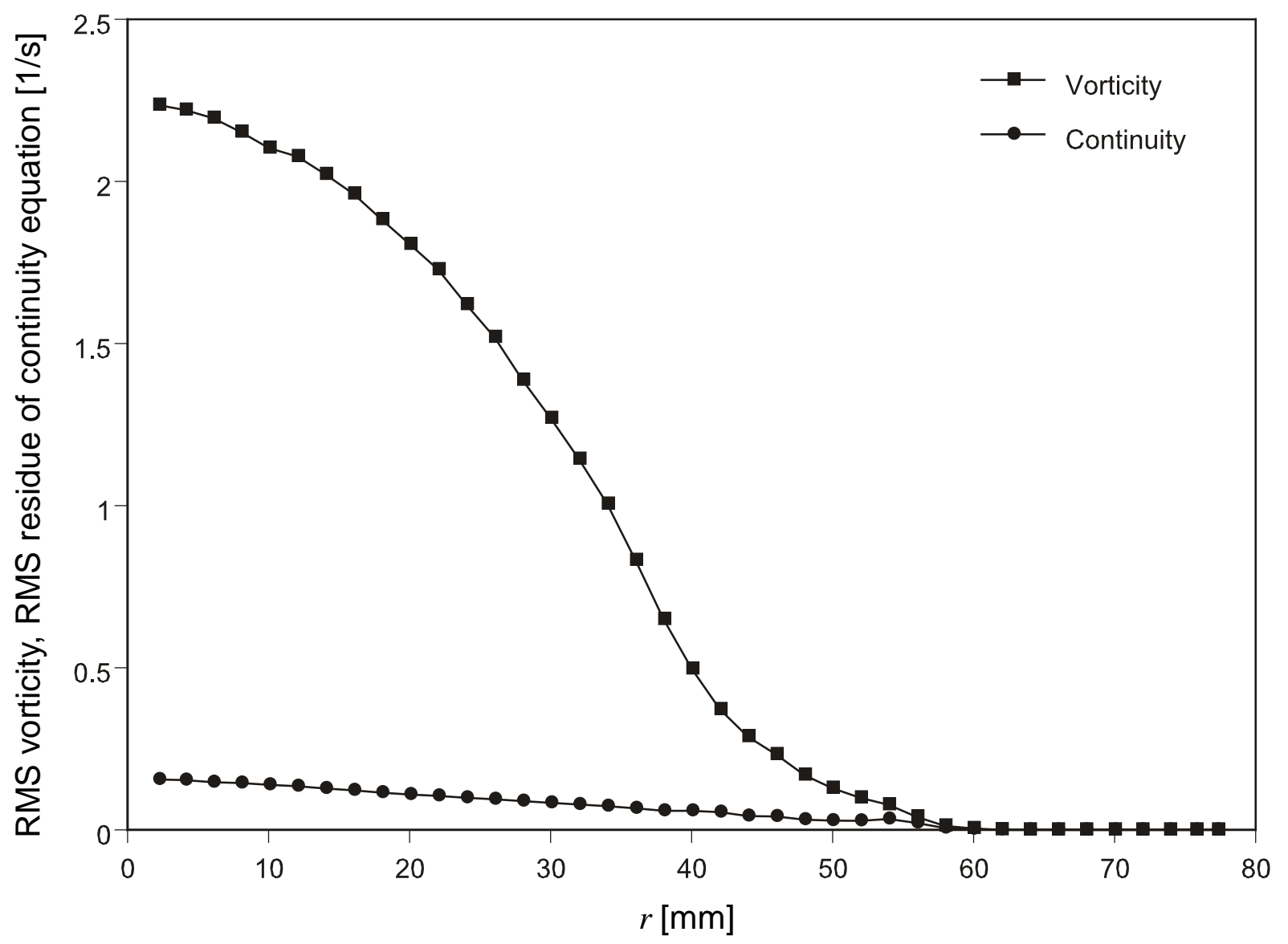

